\title{
DIAGNÓSTICO ULTRA-SONOGRÁFICO DO MOMENTO OVULATÓRIO EM BÚFALAS (Bubalus bubalis) INSEMINADAS NO ESTRO ESPONTÂNEO E INDUZIDO $^{1}$
}

\author{
ULTRASONOGRAPHICAL DIAGNOSIS OF OVULATION IN BUFFALOES (Bubalus bubalis) \\ INSEMINATED IN SPONTANEOUS AND INDUCED OESTRUS
}

\author{
Jorge Damian Stumpfs Diaz ${ }^{2}$ Alexandre Tavares Duarte de Oliveira ${ }^{3}$ \\ Paulo Ricardo Loss Aguiar ${ }^{4}$ José Luiz Rodrigues $^{5}$
}

RESUMO

$O$ objetivo deste experimento foi determinar o intervalo entre o início do estro induzido ou espontâneo $e$ a ovulação em fêmeas bubalinas (Bubalus bubalis) com o auxílio da ultra-sonografia, o que permitirá a determinação de um horário mais apropriado para a I.A. pré-fixada. Nos meses de março a junho, outono no sul do Brasil (época reprodutiva dos bubalinos), 132 fêmeas adultas ciclando foram divididas em 3 grupos experimentais: Grupo A - 53 fêmeas foram tratadas com implante subcutâneo de Norgestomet ou espiral intravaginal contendo progesterona. Na retirada dos dispositivos, foi aplicado $250 \mu \mathrm{g}$ de cloprostenol pela via intra-submucosa vulvar (i.s.m.v.), Grupo B - 48 búfalas foram tratadas com dupla aplicação de $250 \mu \mathrm{g}$ de cloprostenol pela via i.s.m.v. com intervalo de 11 dias e Grupo C 31 búfalas foram consideradas testemunhas, sem tratamento. Todas as búfalas foram inseminadas no momento da observação do maior diâmetro do folículo pré-ovulatório, detectado por ultrasonografia, durante o estro. Após o diagnóstico de prenhez, constatou-se, nos três tratamentos, que houve diferença significativa entre as búfalas prenhes e vazias no período compreendido entre o início do estro até o momento da ovulação $e$ no período entre a I.A.e a ovulação. Os índices de prenhez foram de 41,5\%, 52,1\% e 54,8\% nos grupos $A, B$ e $C$ respectivamente. A variação no intervalo estro-ovulação nas búfalas é uma barreira para a obtenção de taxas de prenhez por I.A. pré-fixada comparáveis à monta natural, tanto no estro induzido através de progesterona e prostaglandina $F_{2}$ alfa como no estro espontâneo.

Palavras-chave: búfalas, progestágeno, prostaglandina $F_{2}$ alfa, ovulação, ultra-sonografia, inseminação artificial.

\section{SUMMARY}

The aim of this experiment was to determine the interval between the beginning of the spontaneous or induced oestrus and the ovulation in females buffaloes using ultrasonography. This will be useful in the determination of the most proper moment for the pre-fixed artificial insemination. In the reproductive season, autumn in the South of Brazil (march-june), 132 clicling females were divided in 3 groups: Group A: 53 females were treated with auricular subcutaneous implant of norgestomet or intravaginal device of progesterone. In the moment that the devices were removed, $250 \mu \mathrm{g}$ of cloprostenol were applied for intravulvar submucosis (ivsm). Group B: 48 buffaloes females were treated twice with $250 \mu \mathrm{g}$ of cloprostenol for ivsm with interval of 11 days. Group C: 31 buffaloes females remained without any treatment (control group). All of them were inseminated in the moment that was observed the biggest diameter of pre-ovulatory follicle determinated by ultrasonography. In the 3 groups, there was significative difference between pregnant and non-pregnant females in the oestrus-ovulation interval and in the A.I.-ovulation interval. The pregnancy rates were $41.5 \%, 52.1 \%$ and $54.8 \%$ in the groups $A, B$ amd $C$, respectively. The variation in the oestrusovulation in buffaloes is the major obstacle to achieve high pregnancy rates using pre-fixed artificial insemination in spontaneous and induced oestrus.

Key words: buffaloes, progestogen, prostaglandin $F_{2}$ alpha, ovulation, ultrasonography, artificial insemination

\section{INTRODUÇÃO}

O rebanho bubalino no Brasil vem crescendo a uma taxa média anual de $12,5 \%$, tendo

\footnotetext{
${ }^{1}$ Parte da Tese de Doutorado apresentada pelo primeiro autor ao Curso de Pós-graduação em Zootecnia, na área de Produção Animal. Universidade Federal do Rio Grande do Sul (UFRGS). Trabalho financiado pela FAPERGS.

${ }^{2}$ Médico Veterinário, MSc, Doutor na área de Produção animal pelo Curso de Pós-graduação em Zootecnia, UFRGS.

${ }^{3}$ Médico Veterinário, MSc, Aluno de Doutorado, Programa de Pós-graduação em Biologia Celular e Molecular, UFRGS.

${ }^{4}$ Médico Veterinário, MSc, Professor da Faculdade de Veterinária, ULBRA, Canoas, RS.

${ }^{5}$ Médico Veterinário, Doutor, Professor Titular, Departamento de Patologia Clínica Veterinária, Faculdade de Veterinária, UFRGS. Bolsista do CNPq, CP15004, 91501-970, Porto Alegre, RS. E-mail: jlr@ orion.ufrgs.br. Autor para correspondência.
} 
alcançado 2,5 milhões de cabeças no país. Apesar do rápido crescimento quantitativo, a melhora qualitativa se dá num ritmo mais lento, devido ao pequeno número de reprodutores com qualidade genética, situação esta que se agrava pela proibição de importar animais de países da Ásia, que apresentam bubalinocultura mais avançada. A multiplicação das aptidões melhoradoras de reprodutores de elite no Brasil, assim como a utilização em larga escala de sêmen importado da Itália ou Bulgária vê-se impedida pela falta de programas práticos e viáveis de inseminação artificial.

Os baixos índices de utilização da IA em bubalinos têm sido atribuídos à dificuldade de detecção do estro na espécie. No entanto, a observação criteriosa com auxílio de rufião (DIAZ, 1988; VALE et al., 1994) tem permitido diagnosticar sinais de estro em até 96,5\% das búfalas observadas (BARUSELLI, 1994).

A sincronização do ciclo estral através da indução do estro com Prostaglandina $F_{2}$ alfa (JAINUDEEN, 1976; KUMARATILLAKE $\boldsymbol{e t}$ al., 1977) ou progestágeno (RAO, 1981; PARGAONKAR et al., 1988) tem sido utilizada, visando à concentração e a detecção dos estros. Na maioria dos experimentos de IA em bubalinos, taxas elevadas de detecção dos estros não correspondem a índices de prenhez considerados economicamente adequados, portanto há outros fatores impedindo a obtenção de taxas de fertilidade através de IA nos bubalinos, semelhantes aos alcançados em outras espécies, como, por exemplo, nos bovinos.

A determinação do momento ovulatório em relação ao início do estro é fundamental para o sucesso da inseminação artificial. As técnicas de diagnóstico da ovulação utilizadas em bubalinos tais como palpação retal e perfil hormonal têm fornecido dados muito variados e pouco precisos. Através da ultrasonografia, será possível obter dados mais acurados quanto ao momento da ovulação, permitindo realizar inseminações em um momento mais adequado no intervalo comprendido entre o início do estro até a ovulação.

O conhecimento do momento ovulatório de búfalas prenhes e vazias após IA no estro espontâneo ou induzido, auxiliará na identificação de intervalos estro-IA e tratamento-IA apropriados para a execução de inseminações com horário pré-fixado.

\section{MATERIAL E MÉTODOS}

O experimento foi realizado em 4 propriedades localizadas no estado do Rio Grande do Sul nos meses de março a junho de 1993 e repetido em
1994, outono no Sul do Brasil, que coincide com a estação reprodutiva dos bubalinos nesta região. Foram utilizadas um total de 132 fêmeas bubalinas adultas (Bubalus bubalis var. bubalis) ou búfalas de rio, incluindo as raças Mediterrâneo, Murrah e Jaffarabadi. Os animais foram divididos ao acaso em 3 grupos experimentais:

\section{Grupo A (tratado com progestágeno $+\mathrm{PGF}_{2} \alpha$ )}

Um grupo de 53 fêmeas, ciclando com média de $2,45 \pm 1,25 \mathrm{ng} / \mathrm{m} \ell$ de progesterona plasmática, teve o estro induzido aleatoriamente através de implante subcutâneo com $3 \mathrm{mg}$ de Norgestomet $^{\mathrm{a}}$ e simultaneamente uma dose de $2 \mathrm{~m} \ell$ com $3 \mathrm{mg}$ de Norgestomet e 3,8mg de Valerato de estradiol, via intramuscular; ou através de dispositivo intravaginal com $1,55 \mathrm{~g}$ de progesterona e $10 \mathrm{mg}$ de benzoato de estradiol $^{\mathrm{b}}$. No momento da retirada do implante $\left(9^{\circ}\right.$ dia) e do dispositivo intravaginal $\left(12^{\circ} \mathrm{dia}\right)$, foi aplicado $1 \mathrm{~m} \ell$ contendo $250 \mu \mathrm{g}$ de cloprostenol ${ }^{\mathrm{c}}$ via i.s.m.v..

\section{Grupo B (tratado com $\mathrm{PGF}_{2} \alpha+\mathrm{PGF}_{2} \alpha$ )}

Um grupo de 48 búfalas, com uma média de $2,52 \pm 1,21 \mathrm{ng} / \mathrm{m} \ell$ de progesterona no plasma, teve o estro induzido com dupla aplicação de $250 \mu \mathrm{g}$ de cloprostenol pela via ismv com intervalo de 11 dias entre uma aplicação e outra.

\section{Grupo C (testemunha)}

Um grupo de 31 búfalas, com uma média de $2,58 \pm 0,66 \mathrm{ng} / \mathrm{m} \ell$ de progesterona plasmática, foi considerado testemunha, sem tratamento.

A partir dos tratamentos, as búfalas foram observadas continuamente, durante 24 horas, para detecção das manifestações do estro com auxílio de rufião com desvio lateral de pênis e deferentectomia, numa proporção de 1 macho por 15 búfalas. A partir da detecção do estro, as búfalas foram examinadas através de ultra-sonografia em intervalos médios de 4 horas até a ovulação (PIERSON \& GINTHER, 1988). O exame ecográfico do folículo préovulatório foi realizado com aparelho de ultrasom Pie Medical Scanner 480 VET, com transdutor linear de $5 \mathrm{Mhz}$. O período de ovulação foi considerado como o intervalo médio (h) entre o último exame, quando o folículo ainda estava presente e o primeiro exame posterior, quando o folículo encontrava-se diminuido no seu tamanho e de contorno irregular ou colapsado (NALBANDOV \& CASIDA, 1942; RODRIGUES DO VALLE $\boldsymbol{e} \boldsymbol{t} \boldsymbol{a l}$., 1994). As imagens do folículo ovulatório antes e depois da ovulação podem ser observadas nas figuras 1,2 e 3 . As búfalas em estro foram insemina- 


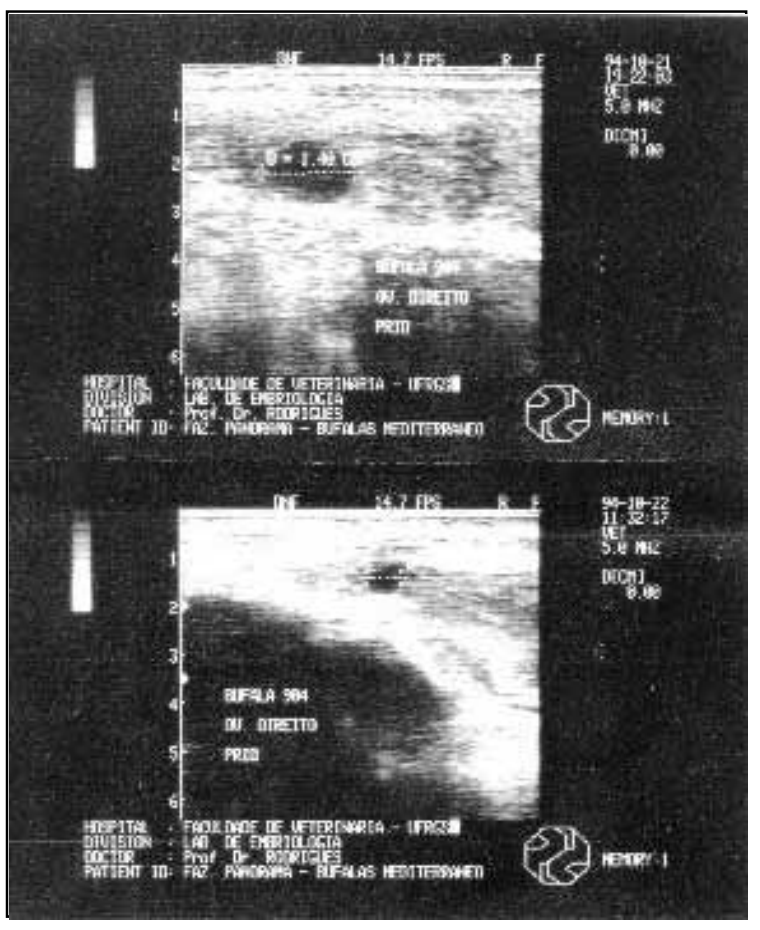

Figura 1 - Imagens ecográficas do folículo ovulatório medido no seu maior diâmetro $(\mathrm{cm})$ do início do estro até a ovulação, numa búfala tratada com PRID (bufala $n^{\circ} 904$, ovário direito).

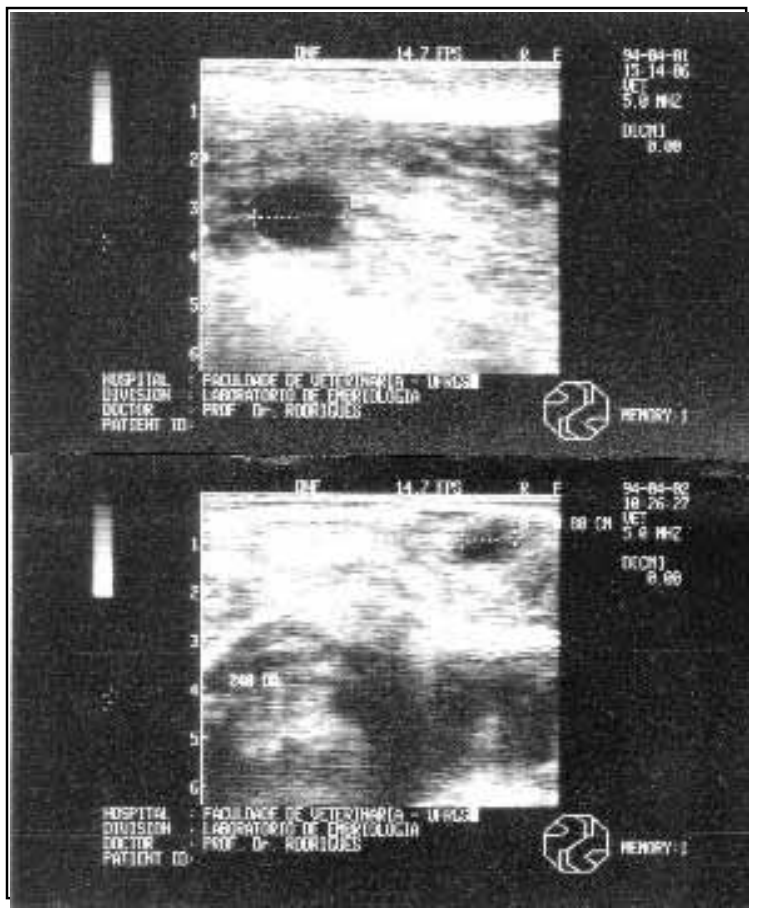

Figura 2 - Imagens ecográficas do folículo ovulatório medido no seu maior diâmetro $(\mathrm{cm})$ do início do estro até a ovulação, numa búfala tratada com dupla aplicação da PgF2alfa (búfala n ${ }^{\circ} 240$, ovário direito).

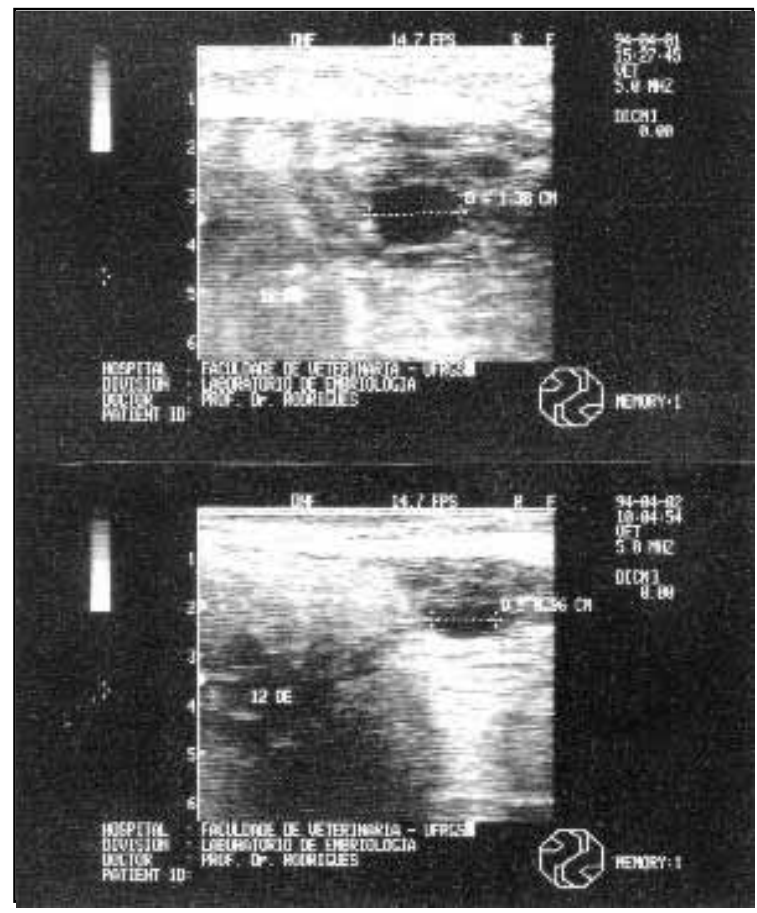

Figura 3 - Imagens ecográficas do folículo ovulatório medido no seu maior diâmetro $(\mathrm{cm})$ do início do estro até a ovulação, numa búfala testemunha (búfala $\mathrm{n}^{\circ} 12$, ovário esquerdo).

inseminadas no momento da observação do maior diâmetro folicular medido em milímetros, através de ultra-sonografia, a partir do início das manifestações do estro. O diagnóstico de prenhez por ultrasonografia foi realizado 40 dias após a inseminação artificial. A análise dos resultados foi feita comparando as búfalas que conceberam com as vazias nos intervalos estro-IA, estro-ovulação e IA-ovulação, em horas. Os dados foram submetidos à análise de variância (ANOVA), através do procedimento GENERAL LINERAL MODELS (GLM) do STATISTICAL ANALYSIS SYSTEM (SAS, 1985), sendo as comparações de médias realizadas pelo teste F.

\section{RESULTADOS E DISCUSSÃO}

Nas taxas de estro e de prenhez (Tabela 1), não houve diferença significativa na incidência de estros entre os grupos tratados com progestágeno + $\mathrm{PgF}_{2} \alpha$ e dupla aplicação de $\operatorname{PgF}_{2} \alpha$ (p>0,05). O índice de estros, no grupo testemunha, foi menor estatisticamente $(p<0,05)$ em relação aos tratados. Essa diferença pode ser atribuída graças à concentração de estros nos grupos tratados, o que facilita a observação e detecção dos mesmos, enquanto, no grupo testemunha, a distribuição dos estros por um período mais longo (21 
Tabela 1 - Incidência de estro e prenhez após a IA no maior diâmetro folicular, nos três tratamentos.

\begin{tabular}{lccccc}
\hline & \multicolumn{3}{c}{ Estro } & \multicolumn{3}{c}{ Prenhez/Estro } \\
\cline { 2 - 6 } Grupos & Total & $\mathrm{N}$ & $\% *$ & $\mathrm{~N}$ & $\%$ \\
\hline A (Progestágeno) & 53 & 53 & $100,00^{\mathrm{A}}$ & 22 & 41,51 \\
B (PgF2 $\alpha+$ PgF2 $\alpha)$ & 50 & 48 & $96,00^{\mathrm{A}}$ & 25 & 52,10 \\
C (Testemunha) & 38 & 31 & $81,57^{\mathrm{B}}$ & 17 & 54,83 \\
Total & 141 & 132 & 93,62 & 64 & 48,48 \\
\hline
\end{tabular}

* Letras diferentes (A, B) indicam diferença significativa entre as proporções a 5\% (Teste de Proporções).

dias) diminuiu a precisão da detecção, principalmente nos estros com manifestações fracas ou muito fracas.

As taxas de prenhez obtidas após a IA no momento de maior diâmetro folicular foram de 41,5\% (22/53), 52,1\% (25/48) e 54,8\% (17/31) nos grupos A, $\mathrm{B}$ e $\mathrm{C}$, respectivamente. Esses índices de fertilidade são similares aos obtidos por SINGH et al. (1987) e DEL REI et al. (1995) com dupla I.A. com búfalas tratadas e BARNABÉ $\boldsymbol{e t}$ al. (1995) com I.A. no final do estro em búfalas testemunhas, podendo ser considerados satisfatórios, tratando-se de taxas obtidas após a realização de uma I.A. no estro.

No intervalo estro-I.A. (Tabela 2) não houve diferença significativa entre as búfalas prenhes e vazias. Dentro do grupo de búfalas vazias, houve diferença significativa $(\mathrm{P}<0,01)$ entre o grupo tratado com progestágeno $+\mathrm{PGF}_{2} \alpha$ e o grupo testemunha $(18,11 \pm 6,37 \times 12,89 \pm 2,46$ horas $)$. Essa diferença poderia ser atribuída a uma falta de precisão na detecção do início do estro nas búfalas do grupo testemunha, o estro pode ter sido diagnosticado algumas horas após o começo das manifestações, enquanto no grupo tratado o sincronismo do início das manifestações estrais facilitou a sua detecção. Mesmo não tendo havido diferença estatisticamente

Tabela 2 - Intervalo médio estro-IA (horas) nas búfalas prenhes e vazias, após a inseminação artifical.

\begin{tabular}{lcccc}
\hline \multirow{2}{*}{ Grupos } & \multicolumn{4}{c}{ Intervalo estro-I.A. (Média \pm DP $)$} \\
\cline { 2 - 5 } & $\begin{array}{c}\text { Progestágeno } \\
(\mathrm{n}=53)\end{array}$ & $\begin{array}{c}\operatorname{PgF} 2 \alpha+\operatorname{PgF} 2 \alpha \\
(\mathrm{n}=48)\end{array}$ & $\begin{array}{c}\text { Testemunha } \\
(\mathrm{n}=31)\end{array}$ & Média Geral \\
\hline $\begin{array}{l}\text { Búfalas prenhes } \\
(\mathrm{n}=64)\end{array}$ & $\begin{array}{c}14,66 \pm 3,89^{\mathrm{A}} \\
(\mathrm{n}=22)\end{array}$ & $\begin{array}{c}13,82 \pm 2,64^{\mathrm{A}} \\
(\mathrm{n}=25)\end{array}$ & $\begin{array}{c}16,76 \pm 6,01^{\mathrm{A}} \\
(\mathrm{n}=17)\end{array}$ & $14,89 \pm 5,76^{\mathrm{A}}$ \\
$\begin{array}{l}\text { Búfalas vazias } \\
(\mathrm{n}=68)\end{array}$ & $\begin{array}{c}18,11 \pm 6,37^{\mathrm{Aa}} \\
(\mathrm{n}=31)\end{array}$ & $\begin{array}{c}16,69 \pm 5,50^{\mathrm{Aab}} \\
(\mathrm{n}=23)\end{array}$ & $\begin{array}{c}12,89 \pm 2,46^{\mathrm{Ab}} \\
(\mathrm{n}=14)\end{array}$ & $16,86 \pm 4,28^{\mathrm{A}}$ \\
\hline
\end{tabular}

-Letras diferentes (A, B), na mesma coluna, indicam diferenças significativas pelo teste $\mathrm{F}$ $(\mathrm{P}<0,01)$.

-Letras diferentes $(a, b)$, na mesma linha, indicam diferenças significativas entre os tratamentos pelo teste $\mathrm{F}(\mathrm{P}<0,01)$. significativa, os resultados sugerem que as inseminações devem ser realizadas em torno das 15 horas após o início do estro nos grupos com estro induzido, e 17 horas, no grupo testemunha, já que a maioria dos autores recomendam inseminações num intervalo amplo de 12 a 24 horas tanto para búfalas com estro induzido pela prostaglandina como para búfalas com a ocorrência de estro espontâneo (LIMA et al., 1981; SINGH et al., 1987; SINGH \& SINGH, 1988; CHOHAN et al., 1992).

Os resultados do experimento corroboram os relatados por AVENELL et al. (1985), que não observaram diferença entre búfalas prenhes e vazias, no intervalo de início do estro ou pico de LH à IA BARUSELLI et al. (1994) também não encontraram diferença estatística $(\mathrm{P}>0,05)$ no índice de concepção de búfalas inseminadas 12, 24 e 36 horas, após o início do estro espontâneo.

Os intervalos estro-ovulação e IA-ovulação (Tabelas 3 e 4) foram significativamente menores $(\mathrm{P}<0,01)$ nas búfalas prenhes em relação às vazias. Isso mostra que, apesar de haver sincronização dos estros dentro de cada grupo tratado, não existe igual grau de sincronização na ovulação. Essa grande variação do período de estro-ovulação foi citada por ZICARELLI et al. (1988), RAUT \& KADU, (1990) e se reflete no intervalo I.A.-ovulação nos grupos de búfalas prenhes e vazias. Como consequiência, as búfalas com uma ovulação mais tardia em relação ao início do estro de 35,01 \pm 6,38, 36,89 \pm 6,22 e 35,07 \pm 6,05 horas para os tratamentos com Progestágeno + $\mathrm{PGF}_{2} \alpha, \quad \mathrm{PGF}_{2} \alpha+\mathrm{PGF}_{2} \alpha$ e testemunhas, respectivamente (Tabela 3 , búfalas vazias) estariam sendo inseminadas em momento inapropriado de $16,90 \pm 4,56,20,19 \pm 6,63$ e 22,18 $\pm 4,48$ horas respectivamente (Tabela 4 , búfalas vazias), ou seja, com um intervalo de tempo muito longo entre a deposição do sêmen no trato genital e a ocorrência da ovulação comprometendo, dessa maneira, a viabilidade dos espermatozóides para fecundar o óvulo.

Como o folículo préovulatório é o maior folículo no pró-estro e estro (DUFUOR $\boldsymbol{e t}$ al., 1971; MATTON et al., 1981; ELWISHY \& GHONEIM, 1995) e tem um crescimento constante, (NALBANDOV \& CASIDA, 1942; MARION et al., 1968), a inseminação artificial neste experimento foi realizada pela observação, com o auxílio ultra-sonográfico, do diâmetro do folículo pré-ovulatório (SINGH et $\boldsymbol{a l} .$, 1987). Levando em consideração 
Tabela 3 - Intervalo médio estro-ovulação (horas) nas búfalas prenhes e vazias após IA, nos três grupos experimentais.

\begin{tabular}{lcccc}
\hline Grupos & \multicolumn{4}{c}{ Intervalo estro-ovulação (Média \pm DP) } \\
\cline { 2 - 5 } & $\begin{array}{c}\text { Progestágeno } \\
(\mathrm{n}=53)\end{array}$ & $\begin{array}{c}\operatorname{PgF} 2 \alpha+\operatorname{PgF} 2 \alpha \\
(\mathrm{n}=48)\end{array}$ & $\begin{array}{c}\text { Testemunha } \\
(\mathrm{n}=31)\end{array}$ & Média Geral \\
\hline $\begin{array}{l}\text { Búfalas prenhes } \\
(\mathrm{n}=64)\end{array}$ & $\begin{array}{c}27,57 \pm 3,88^{\mathrm{A}} \\
(\mathrm{n}=22)\end{array}$ & $\begin{array}{c}26,96 \pm 3,32^{\mathrm{A}} \\
(\mathrm{n}=25)\end{array}$ & $\begin{array}{c}27,97 \pm 5,09^{\mathrm{A}} \\
(\mathrm{n}=17)\end{array}$ & $27,44 \pm 6,23^{\mathrm{A}}$ \\
$\begin{array}{l}\text { Búfalas vazias } \\
(\mathrm{n}=68)\end{array}$ & $\begin{array}{c}35,01 \pm 6,38^{\mathrm{B}} \\
(\mathrm{n}=31)\end{array}$ & $\begin{array}{c}36,89 \pm 6,22^{\mathrm{B}} \\
(\mathrm{n}=23)\end{array}$ & $\begin{array}{c}35,07 \pm 6,05^{\mathrm{B}} \\
(\mathrm{n}=14)\end{array}$ & $35,66 \pm 4,00^{\mathrm{B}}$ \\
\hline
\end{tabular}

- Letras diferentes (A, B), na mesma coluna, indicam diferenças significativas pelo teste $\mathrm{F}(\mathrm{P}<0,01)$

este fato, o intervalo mais longo I.A.-ovulação observado nas búfalas vazias, foi ocasionado pela permanência do folículo ovulatório no seu maior diâmetro, sem a ocorrência de ovulação. $\mathrm{O}$ intervalo mais curto nas búfalas prenhes sugere que a I.A. mais próxima da ovulação, em torno de 12 horas, seria mais eficaz na obtenção de prenhez.

O acompanhamento do folículo préovulatório por ultra-sonografia durante o estro das búfalas, permitiu diagnosticar com precisão o intervalo do início do estro até a ocorrência da ovulação, detectando que há uma variação significativa deste período, quando se compara o grupo das búfalas prenhes com o grupo das fêmeas que ficaram vazias após a IA (Tabela 3). Essa observação permitiu comprovar a ocorrência de uma variação individual no momento ovulatório em relação ao início do estro entre as búfalas, independente dos tratamentos utilizados. Essa variabilidade individual dificulta a determinação de um intervalo de tempo apropriado estro-IA ou tratamento-IA nas búfalas com estro espontâneo ou submetidas à indução do estro, respectivamente. A ocorrência da ovulação com características individuais nas búfalas deve refletir-se nos ciclos estrais subsequentes, sendo cabível considerar que haveria búfalas mais aptas para serem

Tabela 4 - Intervalo médio I.A.-ovulação (horas) nas búfalas prenhes e vazias após I.A., nos três grupos experimentais.

\begin{tabular}{lcccc}
\hline Grupos & \multicolumn{4}{c}{ Intervalo I.A.-ovulação (Média $\pm \mathrm{DP})$} \\
\cline { 2 - 5 } & $\begin{array}{c}\text { Progestágeno } \\
(\mathrm{n}=53)\end{array}$ & $\begin{array}{c}\text { PgF2 } \alpha+\operatorname{PgF} 2 \alpha \\
(\mathrm{n}=48)\end{array}$ & $\begin{array}{c}\text { Testemunha } \\
(\mathrm{n}=31)\end{array}$ & Média Geral \\
\hline $\begin{array}{l}\text { Búfalas prenhes } \\
(\mathrm{n}=64)\end{array}$ & $\begin{array}{c}12,91 \pm 2,66^{\mathrm{A}} \\
(\mathrm{n}=22)\end{array}$ & $\begin{array}{c}13,14 \pm 3,18^{\mathrm{A}} \\
(\mathrm{n}=25)\end{array}$ & $\begin{array}{c}11,20 \pm 1,92^{\mathrm{A}} \\
(\mathrm{n}=17)\end{array}$ & $12,55 \pm 5,68^{\mathrm{A}}$ \\
$\begin{array}{l}\text { Búfalas vazias } \\
(\mathrm{n}=68)\end{array}$ & $\begin{array}{c}16,90 \pm 4,56^{\mathrm{B}} \\
(\mathrm{n}=31)\end{array}$ & $\begin{array}{c}20,19 \pm 6,63^{\mathrm{B}} \\
(\mathrm{n}=23)\end{array}$ & $\begin{array}{c}22,18 \pm 4,48^{\mathrm{B}} \\
(\mathrm{n}=14)\end{array}$ & $19,10 \pm 2,79^{\mathrm{B}}$ \\
\hline
\end{tabular}

- Letras diferentes (A, B), na mesma coluna, indicam diferenças significativas pelo teste $\mathrm{F}(\mathrm{P}<0,01)$. submetidas a programas com uma I.A. pré-fixada.

Os resultados obtidos sugerem a realização de um experimento utilizando-se duas I.A., devendo a segunda ser executada em torno de 8 horas após a primeira, o que corresponderia à diferença média do intervalo estro-ovulação entre prenhes e vazias, para obterem-se melhores índices de fertilidade através de IA.

\section{CONCLUSÕES}

A variabilidade do intervalo início do estro-ovulação nas búfalas não permite a obtenção de taxas mais elevadas de prenhez com uma IA pré-fixada. A IA executada próximo à ovulação, em torno de 12 horas, é eficaz na obtenção de prenhez.

\section{FONTES DE AQUISIÇÃO}

${ }^{\mathrm{a}}$ CRESTAR - Intervet-S.A. - France

${ }^{\mathrm{b}}$ PRID $2 \mathrm{~mm}$ - Sanofi - France

${ }^{c}$ CIOSIN - Pitman-moore do Brasil S.A.

\section{REFERÊNCIAS BIBLIOGRÁFICAS}

AVENELL, J.A., SAEPUDIN, Y., FLETCHER, I.C. Concentrations of LH, Oestradiol-17-B and progesterone in the peripheral plasma of swamp buffalo cows (Bubalus bubalis) around the time of oestrus. Journal Reproduction and Fertility, Cambridge, v.74, p.419-424, 1985.

BARNABÉ, V.H., BARUSELLI, P.S., BARNABÉ, R.C. Implantação de Programas de Inseminação Artificial. In: CONGRESSO BRASILEIRO DE REPRODUÇÃO ANIMAL, 11, 1995, Belo Horizonte, MG. Anais... Belo Horizonte: Colégio Brasileiro de Reprodução Animal, 1995. p.142-155.

BARUSELLI, P.S. Sexual behaviour in buffaloes. In: WORLD BUFFALO CONGRESS, 4, 1994, São Paulo, SP. Proceedings... São Paulo: 1994. V.1, p.158-173.

BARUSELLI, P.S., BARNABÉ, V.H., BARNABÉ, R.C., etal. Artificial insemination in buffalo. In : WORLD BUFFALO CONGRESS, 4., 1994, São Paulo, SP. Proceedings... São Paulo: ABCB, 1994. v.3, p.649-651.

CHOHAN, K.R., CHAUDHRY, R.A., KHAN, N.U., et al. Oestrus behaviour and fertility in normal cycling and oestrus synchronised buffaloes. Indian Journal Dairy Science, New Delhi, v.45, n.11, p.588-590, 1992. 
DEL REI, A.J.M., SILVA, N.Q., SANTOS, M.R.C. Performance reprodutiva em búfalas (Bubalus bubalis) sincronizadas com prostaglandina e inseminadas com sêmen congelado no nordeste do Brasil. In: CONGRESSO BRASILEIRO DE REPRODUÇÃO ANIMAL, 11, 1995, Belo Horizonte, MG. Anais... Belo Horizonte : Colégio Brasileiro de Reprodução Animal, 1995. p 329.

DIAZ, J.D.S. Preparo cirúrgico de rufião em búfalo (Bubalus bubalis). In: CONGRESSO ESTADUAL DE MEDICINA VETERINÁRIA, 10, 1988, Porto Alegre, RS. Anais... Porto Alegre : Sociedade de Veterinária do Rio Grande do Sul, 1988. p.25.

DUFUOR, J., WHITMORE, H.L., GINTHER, O.J., $\boldsymbol{e}$ t $\boldsymbol{a l}$ Identification of the ovulating follicle by its size on different days of the estrous cycle in heifers. Journal of Animal Science, Champaign, v.34, p.85-87, 1971.

EL-WISHY, A.B., GHONEIM, I.M. Ovarian function in buffalo cows with special reference to the accuracy of rectal palpation. Reproduction Domestic Animals, Berlin, v.30, p.39-41, 1995.

JAINUDEEN, M.R. Induction of oestrus and ovulation in buffalo (Bubalus bubalis) using cloprostenol, a synthetic analogue of prostaglandin $\mathrm{F}_{2}$ alfa. Kajian Veterinar, Syngapore, v.8, p.4042, 1976.

KUMARATILLAKE, W.L.J.S., PATHIRAJA, N., PERERA, B.M.A.O., et al. Synchronization of estrus in buffaloes (Bubalus bubalis) using prostaglandin $\mathrm{F}_{2}$ alfa. Research in Veterinary Science, London, v.22, p.380-381, 1977.

LIMA, M.J., GROPP, C.G.L., FONSECA, J., et al. Estudo da viabilidade da I.A. na bubalinocultura a nível do Brasil. In: SIMPÓSIO NACIONAL DE REPRODUÇÃO ANIMAL, 4., 1981, Belo Horizonte, MG. Anais... Belo Horizonte : Colégio Brasileiro de Reprodução Animal, 1981. p.1-10.

MARION, G.B., GIER, M.T., CHOUDARY, J.B Micromorphology of the bovine ovarian follicular system. Journal of Animal Science, Champaign, v.27, p.451-465, 1968.

MATTON, P., ADELAKOUN, V., COUTURE, Y., et al. Growth and replacement of the bovine ovarian follicles during the estrous cycle. Journal of Animal Science, Champaign, v.52, p.813-820, 1981
NALBANDOV, A., CASIDA, L.E. Ovulation and its relation to estrus in cows. Journal of Animal Science, Champaign, v.1, p.189-198, 1942.

PARGAONKAR, D.R., BAKSHI, S.A., JOSHI, S.A., et al. Induction/syncronization of oestrus in buffaloes. In: WORLD BUFFALO CONGRESS, 2, 1988, New Delhi. Proceedings... New Delhi: Indian Council of Agriculture Research, 1988. V.1, p.68.

PIERSON, R.A., GINTHER, O.J. Ultrasonic imaging of the ovaries and uterus in cattle. Theriogenology, New York, v.29, n.1, p.21-37, 1988.

RAO, A.R. Synchronization of oestrus in buffaloes with a progesterone releasing intravaginal device. Veterinary Record, London, v.109, p.123, 1981.

RAUT, N.V., KADU, M.S. Observations on ovulation and its association with fertility in Berari (Nagpuri) buffaloes. Indian Veterinary Journal, Madras, v.67, p.130-132, 1990.

RODRIGUES DO VALLE, E., ENCARNAÇÃO, R.O., SCHENK, J.A.P., et al. Duração do cio e momento de ovulação em vacas nelore. Revista da Sociedade Brasileira de Zootecnia, Viçosa, v. 23, n.5, p.852-858, 1994 .

SAS INSTITUTE.. SAS user's guide: statistic. 5.ed. Cary : SAS, 1985. 956p.

SINGH, G., SINGH, G.B., SHARMA, R.D. Ovulation and fertility after in intravulvo submucous injection of Prosteglandin $\mathrm{F}_{2}$ alpha in subestrous buffaloes. I ndian Journal of Dairy Science, New Delhi, v.40, n.2, p.324-325, 1987.

SINGH, B., SINGH, D. Factors effecting conception rate in buffalo through artificial insemination under field conditions. Indian Journal of Animal Science, New Delhi, v.58, n.7, p.798-799, 1988

VALE, W.G., SOUSA, J.S., RIBEIRO, H.F.L., et al. Preparation of a "Teaser" buffalo bull for use in artificial programs. Buffalo Journal, Bangkok, v.10, n.1, p.75-79, 1994.

ZICARELLI, L., INFASCELLI, F., CAMPANILE, G., et al. Durata del calore (Intertempo tra il suo inizio e la sua fine) ed ovulazione nella búfala in funzione del momento stagionale: Confronto fra estri spontanei ed indotti con alfaprostol. Rivista di Zootechnia e Veterinaria, Milan, v.16, n.1, p.33-55, 1988.

Ciência Rural, v. 31, n. 4, 2001. 